\title{
3-Hydroxyisobutyryl-CoA Hydrolase Deficiency in a Turkish Child with a Novel HIBCH Gene Mutation and Literature Review
}

\author{
Mustafa Kılıça Fatma Kurt-Çolak ${ }^{b}$ \\ ${ }^{a}$ Metabolism Unit, Sami Ulus Children Hospital, Ankara, Turkey; b Medical Genetics Unit, Sami Ulus Children Hospital, \\ Ankara, Turkey
}

\section{Established Facts}

- Newborn and selective screening with tandem mass analyses should include hydroxy-C4-carnitine to diagnose 3-hydroxyisobutyryl-CoA hydrolase deficiency.

\section{Novel Insights}

- A patient with a novel homozygous c.556C>G; p.R186G variant in the HIBCH gene resulted in 3-hydroxyisobutyryl-CoA hydrolase deficiency.

- Hydroxy-C4-carnitine levels may be normal in some cases, mostly in those with milder phenotype.

\section{Keywords}

Developmental delay · Epilepsy $\cdot H I B C H \cdot H I B C H$ deficiency $\cdot$ Valine

\begin{abstract}
3-hydroxyisobutyryl-CoA hydrolase (HIBCH) deficiency (OMIM 250620) is an autosomal recessive inborn error of valine catabolism characterized by severely delayed psychomotor development, progressive neurodegeneration, recurrent metabolic attacks with intercurrent illness, increased lactic acid, cerebral atrophy, and brain lesions in the basal ganglia. $\mathrm{HIBCH}$ gene defect is a very rare organic aciduria and also might cause secondary mitochondrial dysfunction. We report a 12-month-old severely affected female infant with a novel homozygous C.556C > G; p.R186G variant in the $H I B C H$
\end{abstract}

\section{KARGER}

() 2020 S. Karger AG, Basel gene presenting with axial hypotonia, severe developmental delay, and brain lesions in the basal ganglia and provide an overview of the literature. When suspected, newborn and selective screening with tandem mass analyses should include hydroxy-C4-carnitine to diagnose this disorder. However, in some cases, mostly in those with milder phenotype, diagnosis may be missed due to normal hydroxy-C4 carnitine levels.

(c) 2020 S. Karger AG, Basel

Inborn errors of valine degradation include isobutyrylCoA dehydrogenase deficiency, short-chain enoyl-CoA hydratase deficiency, 3-hydroxyisobutyryl-CoA hydrolase $(\mathrm{HIBCH})$ deficiency, 3-hydroxyisobutyryl-CoA dehydrogenase (HIBADH) deficiency, and methylmalonic 


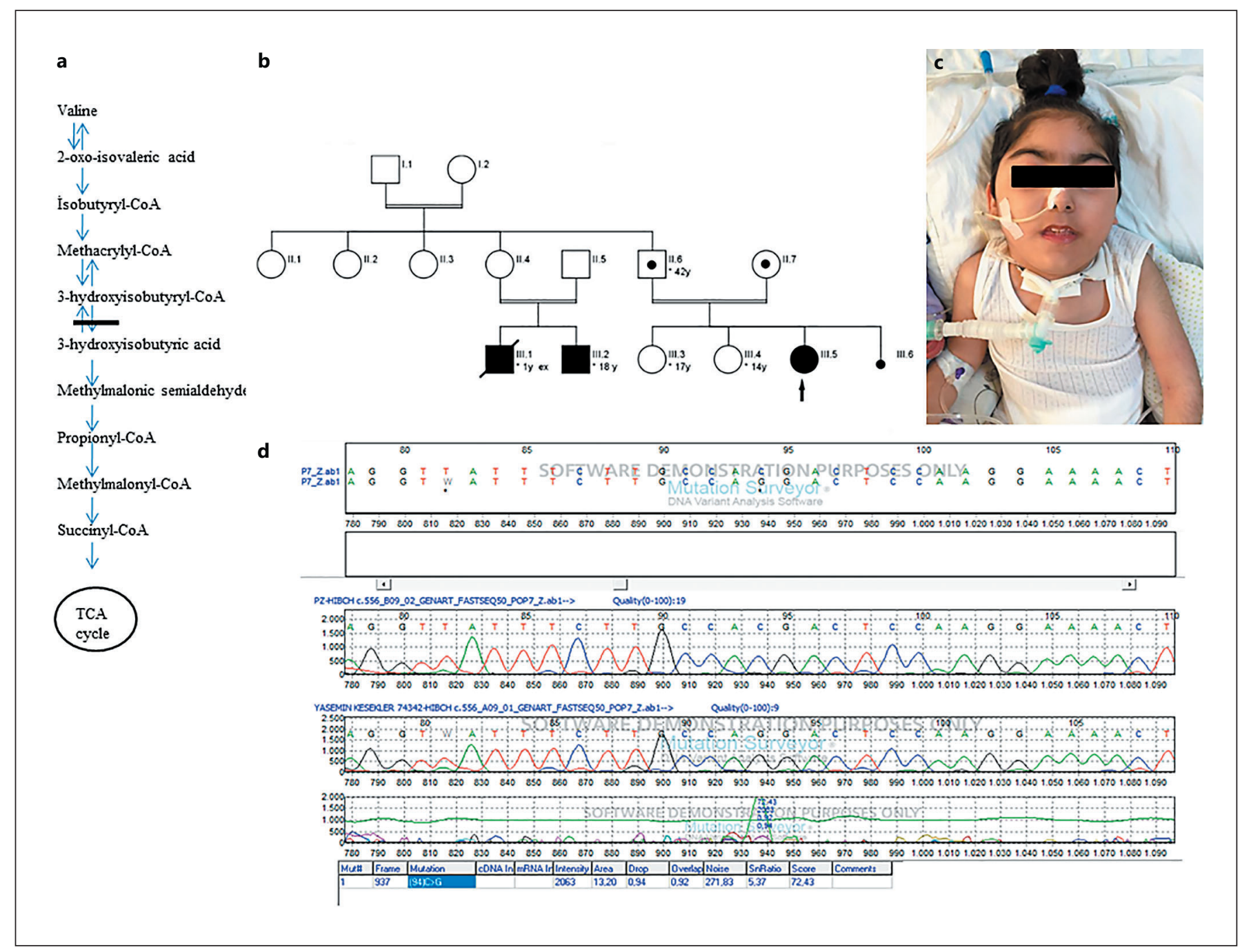

Fig. 1. a Valine oxidation pathway. Solid bar shows 3-hydroxyisobutyryl-CoA hydrolase. b The pedigree of the family with HIBCH deficiency. c Photograph of the patient at age 5 years 3 months. d Sequence electropherograms of the $\mathrm{HIBCH}$ gene indicating a homozygous c.556C>G; p.R186G variant.

semialdehyde dehydrogenase deficiency [Wanders et al., 2012; Peters et al., 2014, 2015]. HIBCH is a mitochondrial enzyme that catalyses the fifth step of valine catabolism, the conversion of 3-hydroxyisobutyryl-CoA to 3-hydroxyisobutyrate and also acts on 3-hydroxypropionyl$\mathrm{CoA}$, giving it a dual role in a secondary pathway of propionate metabolism (Fig. 1a) [Wanders et al., 2012; Peters et al., 2014, 2015]. HIBCH (or deacylase) deficiency (OMIM 250620) is an autosomal recessive inborn error of valine catabolism characterized by congenital malformations, mild dysmorphic facial features, microcephaly, severely delayed psychomotor development, hypotonia, ataxia, dysarthria, spasticity, dystonia, optic nerve atrophy, poor feeding, seizures starting in infancy, developmental regression, episodes of ketoacidosis and encephalopathy, increased lactic acid, and Leigh-like changes in the basal ganglia. It is a rare cerebral organic aciduria and may cause a combined deficiency of multiple mitochondrial respiratory chain enzymes and pyruvate dehydrogenase [Ferdinandusse et al., 2013]. The highly reactive metabolites methacrylyl-CoA and acryloyl-CoA accumulate and are probably responsible for the brain pathology [Peters et al., 2014]. Methacrylyl-CoA is a highly reactive compound which easily reacts with free sulfhydryl groups, 
potentially interfering with mitochondrial enzymes and depleting mitochondrial pools of cysteine, glutathione, thioredoxin, CoA, or lipoic acid [Ferdinandusse et al., 2013]. It was first described in 1982, and its gene was cloned in 1996 [Brown et al., 1982; Hawes et al., 1996; Loupatty et al., 2007]. The HIBCH gene maps to chromosome 2 q32.2 and has an open reading frame of $1,161 \mathrm{bp}$, encoding a protein of 386 amino acid residues with a molecular weight of $43 \mathrm{kDa}$. Its incidence was estimated at approximately 1:551,545 in Europeans and 1:127,939 in South Asian individuals [Stiles et al., 2015]. To date, 25 patients from 19 families have been reported [Brown et al., 1982; Loupatty et al., 2007; Ferdinandusse et al., 2013; Yamada et al., 2014; Reuter et al., 2014, 2017; Peters et al., 2015; Soler-Alfanso et al., 2015; Stiles et al., 2015; Zhu et al., 2015; Charng et al., 2016; Schottmann et al., 2016; Tan et al., 2018; Yang et al., 2018; Candelo et al., 2019; Karimzadeh et al., 2019; Xu et al., 2019; D'Gama et al., 2020]. Elevated levels of hydroxy-C4-carnitine in dried blood spots or plasma and measurement of the unusual amino acids S-(2-carboxypropyl) cysteine (SCPC) and S-(2-carboxypropyl) cysteamine (SCPCM) as well as their carnitine esters in urine by tandem mass spectrometry is effective for initial screening [Peters et al., 2015]. Plasma and cerebrospinal fluid lactate levels may be normal or elevated. A definitive diagnosis of HIBCH deficiency can be made by measurement of $\mathrm{HIBCH}$ enzymatic activity in patient tissues and/or molecular analysis of the HIBCH gene. Symmetrical involvement of the basal ganglia, especially globi pallidi and acute changes suggestive of cytotoxic edema in acute attacks can be seen on brain MRI [Stiles et al., 2015; Xu et al., 2019; D'Gama et al., 2020]. Although there is no effective treatment, low-protein (low-valine diet), highcarbohydrate diet, and L-carnitine, $\mathrm{N}$-acetylcysteine, and mitochondrial cocktail treatments have been suggested [Loupatty et al., 2007; Yamada et al., 2014; Peters et al., 2015; Soler-Alfonso et al., 2015; D'Gama et al., 2020]. We aimed to present clinical and biochemical findings as well as molecular genetic data in a new case of a Turkish girl with HIBCH deficiency and to review the literature.

\section{Case Report}

A 1-year-old female infant was admitted to our clinic due to being unable to sit independently. She had achieved head control at 3 months and could sit with support at 6 months. Her developmental delay was observed after 9 months, still being unable to sit unaided. She was born as a third child of healthy, consanguineous Turkish parents. She was born at term (40 weeks) via normal spontaneous vaginal delivery with $3,240 \mathrm{~g}$ birth weight. Intrauterine period and delivery were uneventfully. Parents were second cousins. She had 2 healthy siblings. One cousin had died of neurological problems at 1 year of age, and another cousin had epilepsy and developmental delay without diagnosis at the age of 18 years (Fig. 1b). Physical examination showed axial hypotonia, developmental delay, bilaterally increased deep tendon reflexes, and positive Babinsky sign. She could not sit without support and spoke no discernible words (Fig. 1c). Laboratory findings showed normal ammonia levels, whereas serum lactate $(26.7 \mathrm{mg} / \mathrm{dL}, \mathrm{N}: 4.5-19.8)$ and pyruvate $(1.1 \mathrm{mg} / \mathrm{dL}, \mathrm{N}: 0.3-0.9)$ levels were mildly elevated. Plasma and/or serum creatinine, uric acid, homocysteine, vitamin B12, creatine kinase, complete blood count, and liver, kidney and thyroid function tests were normal. Metabolic investigations including plasma and urine quantitative amino acid analyses, biotinidase activity, and urinary organic acid analyses were normal (SCPC and SCPCM were not available and not included in the analyses). Repetitive acylcarnitine analyses with mass spectrometry showed increased plasma hydroxy-C4-carnitine [(3-OH-butyryl): 3.84 and 3.6 (N: 0-0.48 $\mu \mathrm{mol} / \mathrm{L}$ )]. Short-chain 3-hydroxyacyl-CoA dehydrogenase (SCHAD) deficiency, HIBADH deficiency, and $\mathrm{HIBCH}$ deficiency were suspected. Abdominal and renal Doppler ultrasound findings were normal. Echocardiography showed interventricular septal hypertrophy. Ophthalmological examination showed exotropia and bilaterally pale optic discs. Reye-like syndrome with hypoglycemia, metabolic and lactic acidosis, elevated liver enzymes, hyperuricemia, and encephalopathy was observed following acute gastroenteritis at the age of 15 months. Acute phase reactants and cultures (urine, blood, cerebrospinal fluid) were normal. Symptomatic treatment with intravenous fluids with bicarbonate and high glucose infusion rate $(8 \mathrm{mg} /$ $\mathrm{kg} / \mathrm{min}$ ) were given. Brain MRI performed at age 15 months during this acute attack detected cerebral atrophy, symmetric signal hyperintensity and swelling in basal ganglia as well as increased periventricular signal intensities. Lesions showed restricted diffusion. These lesions were suggestive of mitochondrial disorders, especially Leigh syndrome or organic acidemia. Mitochondrial cocktail (thiamine, riboflavin, coenzyme Q10, vitamin C, vitamin E, biotin, L-carnitine) therapy was given. N-Acetylcysteine 100-150 $\mathrm{mg} / \mathrm{kg} / 24-\mathrm{h}$ infusion was also added. Encephalopathy resolved and her clinical condition improved. However, she had no spontaneous respiration or swallowing. She was discharged from the clinic receiving home mechanical ventilation and nasogastric tube at age 17 months. At 18 months, recurrent generalized tonic, clonic, and myoclonic seizures started; antiepileptic drugs were given. Electroencephalography showed epileptic discharges. Seizures continued in spite of drug treatment. Clinical exome panel encompassing 4,500 disorders detected a homozygous c.556C $>\mathrm{G}$; p.R186G variant in the $H I B C H$ gene (NM_014362.3), and she was diagnosed with HIBCH deficiency (Fig. 1d). Both of her parents were found to be heterozygous for this variant. Her affected cousins probably may have had the same disorder, but the parents did not give permission to metabolic and genetic testing. Low-protein, high-carbohydrate diet was started in addition to mitochondrial cocktail. Unfortunately, $\mathrm{HIBCH}$ enzyme activity and mitochondrial complex activities of the oxidative phosphorylation have not been studied. The patient had recurrent hospitalizations due to urinary tract infections, aspiration, and pneumonia. She is 6 years old now, and the treatment outcome is poor with oxygen dependence and severe developmental delay. She has a nasogastric tube and tracheostomy with home mechanical ventilatation. 


\section{Materials and Methods}

Exome capture and sequencing was performed for sample preparation, and exonic DNA was captured using the Clinical Exome Solution (SOPHiA GENETICS). Illumina NextSeq 500/550 High Output Kit v2.5 was used for paired-end cluster generation and the v2.5 reagent kit for PE $2 \times 150$ sequencing of the postcapture libraries with the NextSeq 500/550 system (Illumina Inc., San Diego, CA, USA). All procedures were carried out according to the manufacturer's instructions with slight optimizations. Base calling and image analyses were done using Sophia DDM ${ }^{\mathrm{TM}}$ platform. Sanger sequencing of the patient and her parents confirmed mutations/variants related to her phenotype. Systematic literature searching with Pubmed was done using the following terms: "HIBCH”, "HIBCHD”, "HIBCH deficiency", "3-hydroxyisobutyryl-CoA hydrolase deficiency."

\section{Results and Discussion}

Very few patients with $H I B C H$ gene defect have been described in the literature. A comprehensive data set with published reports on $\mathrm{HIBCH}$ deficiency patients together with our patient are summarized in online supplementary Table 1 (see www.karger.com/doi/10.1159/000508728). So far, including the patient presented herein, 24 different mutations in 20 families with 26 affected patients have been reported. Male to female ratio was 14/11 (one not available). $44 \%$ of these families with $\mathrm{HIBCH}$ gene defect had consanguineous marriages (8/18 families, 2 of them not available). Most of the patients had Asian or Middle Eastern origin, rarely Caucasian or South American. Six of 26 patients from 3 unrelated families were of Turkish origin ( $23 \%$ of the patients, $15 \%$ of the families). Age at onset of clinical findings ranged from birth to 48 months (24/24 patients $=100 \%, 2$ not available $)$ and in most cases $(18 / 24$ patients $=75 \%, 2$ not available $)$ before 1 year of age. Seven of 26 patients $(27 \%)$ died in early childhood at the time of publication. Nearly all patients had severe neurological deficits, except for those in 3 families reported in the literature [Schottmann et al., 2016; Xu et al., 2019]. In one report, 5 Turkish patients from 2 unrelated families with a homozygous c.913A>G; p.T305A mutation in the $\mathrm{HIBCH}$ gene were seen in adolescence and adulthood. Onset of their clinical findings was later than 1 year, and they had a milder phenotype than others [Schottmann et al., 2016]. Interestingly, in this report, in 2 patients from the same family, hydroxyl-C4-carnitine levels were normal. Additionally, in another report, a milder clinical presentation with only exercise-induced dystonia was observed in a 6-year-old Chinese girl with compound heterozygous c.1027C $>$ G; p.H343D/c.383T >A; p.V128D mutations in $H I B C H$ [Xu et al., 2019]. Hydroxy-C4-carnitine level was also normal in this patient. Among the reported patients with known hydroxy-C4-carnitine levels, these were found to be increased in the all of the other severely affected patients, except one [D'Gama et al., 2020]. HIBCH enzyme activities were low in the majority of the reported patients. Mitochondrial complex activities, pyruvate dehydrogenase activities, and the quantity of mtDNA in tissues were found to be normal or decreased. All mutations were different from each other and specific to families, except for c.913A $>G$, which was seen in homozygous state in 2 unrelated Turkish families and in heterozygous state in one Iranian family. c.808A>G, which was seen in homozygous state in one Colombian patient and in heterozygous state in one unrelated other Colombian patient. So far, there seem to be no race-specific mutations. In the literature, patients carrying truncating mutations tended to have more severe phenotypes compared with missense mutations [Tan et al., 2018; D'Gama et al., 2020]. Additionally, it was also discussed that missense mutations around the catalytic site of the enzyme (p.A96D, p.Y122C, p.G317E, p.G345S), the substrate- (3-hydroxyisobutyrate) binding pouch and cofactor- (pentahydroxyflavone) binding site cause a more severe phenotype than mutations in the periphery of the enzyme (p.R66W, p.A137V, p.T305A) [Schottmann et al., 2016]. In our patient, the p.R186G variant resulted in a severe phenotype. This variant is predicted to be "probably damaging" by PolyPhen-2 (0.99) and SIFT (1.0), and "disease causing" by Mutation Taster. It is classified as a variant of unknown significance according to American College of Medical Genetics and Genomics (http://wintervar.wglab.org/) (ACMG) 2015 guidelines (PM2, PP3, PP5). HIBCH protein molecules form a homocomplex, and $\operatorname{Arg} 186$ of HIBCH is located at the trimer interface of HIBCH molecules in the crystal structure model (https:// www.ncbi.nlm.nih.gov/Structure/pdb/3BPT). Therefore, the p.R186G variant was predicted to affect the formation of the homocomplex. In general, all of the reported mutations, except homozygous p.T305A and compound heterozygous p.H343D/p.V128D, seem to cause a severe phenotype. However, it is too early to make genotype-phenotype correlations. The diversity and spectrum of the clinical findings in $\mathrm{HIBCH}$ gene defects will probably expand, and a genotype-phenotype correlation might become evident as reported cases increase. Most of the patients described in the literature have brain MRI findings resembling Leigh syndrome, such as bilateral basal ganglia involvement and atrophy of the white matter. During or following acute exacerbations, symmetrical T2-weighted 
hyperintensities with restricted diffusion were observed in the basal ganglia, especially in the globus pallidus. With years of progression, cystic encephalomalasia, basal ganglion atrophy and necrosis may ensue [Stiles et al., 2015; D'Gama et al., 2020]. Our findings on brain MRI were similar to the literature, showing symmetrical bilateral lesions in the basal ganglia, suggestive of a progressive mitochondrial disorder, especially Leigh syndrome (OMIM 256000 ) or organic acidemia. Clinical exome panel established the diagnosis. HIBCH deficiency is also reported as one of the secondary causes of Leigh syndrome [Ferdinandusse et al., 2013; Reuter et al., 2014; Yamada et al., 2014; Soler-Alfonso et al., 2015; Stiles et al., 2015].

HIBCH deficiency can be detected by newborn screening cards. This disorder may be underdiagnosed and more prevalent than currently appreciated [Stiles et al., 2015]. Screening programs should include hydroxy-C4carnitine to detect these patients. However, we observed that hydroxy-C4-carnitine levels might be normal in a few cases, mostly in milder phenotypes [Schottmann et al., 2016; Xu et al., 2019; D’Gama et al., 2020]. HIBCH deficiency should be suspected in patients with unexplained developmental delay and epilepsy together with persistently elevated plasma hydroxy-C4-carnitine. The differential diagnoses for elevated hydroxy-C4-carnitine are known as $\mathrm{HIBCH}$ deficiency (HIBCH affected) and SCHAD deficiency $(H A D H)$. Patients with SCHAD deficiency clinically differ from $\mathrm{HIBCH}$ deficiency and suffer from hyperinsulinism and hypoglycemia. This is not to be confused with elevated levels of C4-carnitine, which are observed in patients with isobutyryl-CoA dehydrogenase deficiency (ACAD8), short-chain acyl-CoA dehydrogenase deficiency (ACADS), and ethylmalonic encephalopathy (ETHE1). Mitochondrial disorders including pyruvate dehydrogenase complex deficiency, biotin-thiamine responsive basal ganglia disease, pyruvate carboxylase deficiency, and short-chain enoyl-CoA hydratase deficiency were also discussed in the differential diagnosis of HIBCH deficiency [Stiles et al., 2015]. In our patient, clinical exome panel was preferred because more than 3 inherited disorders were suspected (SCHAD deficiency, HIBADH deficiency, HIBCH deficiency, and mitochondrial disorder, especially Leigh syndrome). These cases illustrate the clinical utility of clinical exome panel and also acylcarnitine analyses in metabolic disorders.

There is no treatment currently available. Low-protein (low valine) and a high-carbohydrate diet were recommended [Yamada et al., 2014; Soler-Alfonso et al., 2015; Yang et al., 2018; Candelo et al., 2019; Xu et al., 2019]. Lcarnitine for the release of CoA and increasing the elim- ination of 3-hydroxyisobutyryl-CoA as urinary 3-hydroxyisobutyrylcarnitine and $\mathrm{N}$-acetylcysteine for maintaining glutathione reserves were suggested [Loupatty et al., 2007]. The antioxidants ascorbate and $\mathrm{N}$-acetylcysteine, pantothenate, and cysteamine might improve mitochondrial function in HIBCH deficiency patients [Loupatty et al., 2007; Peters et al., 2015]. We continued the low-protein and high-carbohydrate diet as well as L-carnitine and mitochondrial cocktail supplementation. Clinical improvement was achieved with this treatment, especially in an acute attack. This observation was similar to other organic acidemias that $\mathrm{HIBCH}$ deficiency is also an intoxication-type metabolic disorder and may benefit from treatment in acute attacks caused by catabolic stress conditions such as infections, fever, or trauma. Despite 6 months of therapy, no clinical improvements were observed in our patient as she was already severely affected, but the alertness of the patient did improved.

In conclusion, persistently elevated levels of hydroxy-C4-carnitine through blood spot acylcarnitine analyses should be carefully interpreted and used as a first tier screening test for patients suspected of this condition in newborn screening programs using tandem mass spectrometry. However, in some cases, mostly with a milder phenotype, the diagnosis may be missed due to normal hydroxy-C4 carnitine levels. Newborn screening may help to make early diagnosis and early treatment with a better outcome.

\section{Acknowledgment}

We sincerely thank the family of the patient for participating in this study.

\section{Statement of Ethics}

All procedures performed in this study were in accordance with the ethical standards of the institutional and/or national research committee and with the 1964 Helsinki declaration and its later amendments or comparable ethical standards. Written informed consent for participation and publication of clinical data was obtained from the family.

\section{Disclosure Statement}

The authors declare no conflicts of interest. 


\section{Funding Sources}

The funding organization(s) played no role in the study design or writing, in the collection, analysis, interpretation of data, or in the decision to submit the report for publication.

\section{Author Contributions}

The authors are responsible for the entire content of the report. They read and approved the final version and submission of the manuscript. M.K. did the general evaluation of the patient, analyses of biochemical data, and drafting the article. F.K.Ç. performed molecular and data analyses of the patient.

\section{References}

Brown GK, Hunt SM, Scholem R, Fowler K, Grimes A, et al: Beta-hydroxyisobutyryl coenzyme A deacylase deficiency: a defect in valine metabolism associated with physical malformations. Pediatrics 70:532-538 (1982).

Candelo E, Cochard L, Caicedo-Herrera G, Granados AM, Gomez JF, et al: Syndromic progressive neurodegenerative disease of infancy caused by novel variants in $H I B C H$ : report of two cases in Colombia. Intractable Rare Dis Res 8:187-193 (2019).

-Charng WL, Karaca E, Coban Akdemir Z, Gambin T, Atik MM, et al: Exome sequencing in mostly consanguineous Arab families with neurologic disease provides a high potential molecular diagnosis rate. BMC Med Genomics 9:42 (2016).

D'Gama AM, Brucker WJ, Zhang T, Gubbels CS, Ferdinandusse S, et al: A phenotypically severe, biochemically "silent" case of $\mathrm{HIBCH}$ deficiency in a newborn diagnosed by rapid whole exome sequencing and enzymatic testing. Am J Med Genet A 182:780-784 (2020).

-Ferdinandusse S, Waterham HR, Heales SJ Brown GK, Hargreaves IP, et al: $\mathrm{HIBCH}$ mutations can cause Leigh-like disease with combined deficiency of multiple mitochondrial respiratory chain enzymes and pyruvate dehydrogenase. Orphanet J Rare Dis 8:188 (2013).

Hawes JW, Jaskiewicz J, Shimomura Y, Huang B, Bunting J, et al: Primary structure and tissuespecific expression of human beta-hydroxyisobutyryl-coenzyme A hydrolase. J Biol Chem 271:26430-26434 (1996).
Karimzadeh P, Saberi M, Sheidaee K, Nourbakhsh M, Keramatipour M: 3-Hydroxyisobutyryl-CoA hydrolase deficiency in an Iranian child with novel $H I B C H$ compound heterozygous mutations. Clin Case Rep 7: 375-380 (2019).

Loupatty FJ, Clayton PT, Ruiter JP, Ofman R, Ijlst $\mathrm{L}$, et al: Mutations in the gene encoding 3-hydroxyisobutyryl-CoA hydrolase results in progressive infantile neurodegeneration. Am J Hum Genet 80:195-199 (2007).

- Peters H, Buck N, Wanders R, Ruiter J, Waterham $\mathrm{H}$, et al: ECHS1 mutations in Leigh disease: a new inborn error of metabolism affecting valine metabolism. Brain 137:2903-2908 (2014).

- Peters H, Ferdinandusse S, Ruiter JP, Wanders RJ, Boneh A, Pitt J: Metabolite studies in $\mathrm{HIBCH}$ and ECHS1 defects: implications for screening. Mol Genet Metab 115:168-173 (2015).

Reuter MS, Sass JO, Leis T, Köhler J, Mayr JA, et al: $\mathrm{HIBCH}$ deficiency in a patient with phenotypic characteristics of mitochondrial disorders. Am J Med Genet A 164A:3162-3169 (2014).

Reuter MS, Tawamie H, Buchert R, Hosny Gebril $\mathrm{O}$, Froukh T, et al: Diagnostic yield and novel candidate genes by exome sequencing in 152 consanguineous families with neurodevelopmental disorders. JAMA Psychiatry 74:293299 (2017).

-Schottmann G, Sarpong A, Lorenz C, Weinhold $\mathrm{N}$, Gill E, et al: A movement disorder with dystonia and ataxia caused by a mutation in the $H I B C H$ gene. Mov Disord 31:1733-1739 (2016).

- Soler-Alfonso C, Enns GM, Koenig MK, Saavedra $\mathrm{H}$, Bonfante-Mejia E, Northrup H: Identification of $\mathrm{HIBCH}$ gene mutations causing autosomal recessive Leigh syndrome: a gene involved in valine metabolism. Pediatr Neurol 52:361-365 (2015).
- Stiles AR, Ferdinandusse S, Besse A, Appadurai V, Leydiker KB, et al: Successful diagnosis of $\mathrm{HIBCH}$ deficiency from exome sequencing and positive retrospective analysis of newborn screening cards in two siblings presenting with Leigh's disease. Mol Genet Metab 115:161-167 (2015).

Tan H, Chen X, Lv W, Linpeng S, Liang D, Wu L: Truncating mutations of $\mathrm{HIBCH}$ tend to cause severe phenotypes in cases with $\mathrm{HIBCH}$ deficiency: a case report and brief literature review. J Hum Genet 63:851-855 (2018).

-Wanders RJ, Duran M, Loupatty FJ: Enzymology of the branched-chain amino acid oxidation disorders: the valine pathway. J Inherit Metab Dis 35:5-12 (2012).

Xu Y, Zhang J, Yu K, Feng F, Sun X, et al: A therapeutic regimen for 3-hydroxyisobutyrylCoA hydrolase deficiency with exercise-induced dystonia. Eur J Paediatr Neurol 23: 755-759 (2019).

- Yamada K, Naiki M, Hoshino S, Kitaura Y, Kondo $\mathrm{Y}$, et al: Clinical and biochemical characterization of 3-hydroxyisobutyryl-CoA hydrolase $(\mathrm{HIBCH})$ deficiency that causes Leighlike disease and ketoacidosis. Mol Genet Metab Rep 1:455-460 (2014).

Yang HY, Wu LW, Deng XL, Yin F, Yang LF: Diagnosis and treatment of 3-hydroxyisobutyryl-CoA hydrolase deficiency: a case report and literature review (in Chinese). Zhongguo Dang Dai Er Ke Za Zhi 20:647-651 (2018).

Zhu H, Bao X, Zhang Y: 3-Hydroxy-isobutyrylCoA hydrolase deficiency in a child with Leigh-like syndrome and literature review (in Chinese). Zhonghua Er Ke Za Zhi 53:626-630 (2015). 